\title{
Downregulation of mouse CCR3 by lentiviral shRNA inhibits proliferation and induces apoptosis of mouse eosinophils
}

\author{
XIN-HUA ZHU ${ }^{1}$, BING LIAO ${ }^{2}$, YI XU ${ }^{1}, \mathrm{KE} \mathrm{LIU}^{1}$, YUN HUANG ${ }^{1}$, QUAN-LONG HUANG ${ }^{1}$ and YUE-HUI LIU $^{1}$ \\ ${ }^{1}$ Department of Otolaryngology Head and Neck Surgery, The Second Affiliated Hospital of Nanchang University; \\ ${ }^{2}$ Department of Head and Neck Surgery, The Tumor Hospital of Jiangxi Province, Nanchang, Jiangxi 330006, P.R. China
}

Received August 31, 2015; Accepted August 24, 2016

DOI: $10.3892 / \mathrm{mmr} .2016 .6085$

\begin{abstract}
RNA interference has been considered as an effective gene silencing method in basic and preclinical investigations. The aims of the present study were to construct a lentiviral vector expressing a short hairpin RNA (shRNA) targeting the murine $\mathrm{CC}$ chemokine receptor 3 (mCCR3), and to investigate its effects on the proliferation and apoptosis of mouse eosinophils. A recombinant lentiviral vector expressing four fragments of mouse CCR3 shRNA (pLVX-mCCR3-1+2+3+4-shRNA) was constructed using subcloning techniques. This novel lentivirus was then packaged into $293 \mathrm{~T}$ cells by co-transduction with plasmids, including Baculo p35, pCMV R8.2 and VSV. The interference effects of the vector were verified using polymerase chain reaction (PCR) and western blot analyses. The effects of the interference on the proliferation and apoptosis of mouse eosinophils were investigated using 3-(4,5-dimethylthiazol-2-yl)5-(3-carboxymethoxyphenyl)-2-(4-sulfophenyl)-2H-tetrazolium and terminal deoxynucleotidyl transferase dUTP nick end labeling methods, respectively. The results of the PCR and western blot analyses confirmed that the novel recombinant vector, pLVX-mCCR3-1+2+3+4-shRNA, had high efficiency in inhibiting the mRNA and protein expression levels of mCCR3 in mouse eosinophils. The downregulation of mCCR3 significantly inhibited proliferation of the eosinophils. Furthermore, the present study found that the downregulation of mCCR3 significantly promoted apoptosis of the eosinophils. Therefore, the downregulation of mCCR3 led to the inhibition of proliferation and induction of apoptosis in mouse eosinophils. The predominant characteristics of allergic rhinitis are eosinophil infiltration and release of inflammatory mediators, which appear in a variety of clinical
\end{abstract}

Correspondence to: Dr Yue-Hui Liu, Department of Otolaryngology Head and Neck Surgery, The Second Affiliated Hospital of Nanchang University, 1 Minde Road, Nanchang, Jiangxi 330006, P.R. China

E-mail: liuyuehuiclark@21cn.com

Key words: murine CC chemokine receptor 3, eosinophil, short hairpin RNA, apoptosis manifestations. The results of the present study indicate that mCCR3 silencing may serve as a putative approach for the treatment of allergic rhinitis.

\section{Introduction}

Allergic rhinitis (AR) is a common global health problem, which has a severe affect on daily life. The morbidity rate of AR has increased in previous decades and affects $10-20 \%$ of the population in western countries (1). A report from 2014 from Beijing Tongren Hospital on the prevalence of allergic rhinitis in China showed that the morbidity rate of AR in China is also increasing, as is the prevalence of a 'western'-type lifestyle (2).

AR has been identified as a chronic inflammatory disease of the nasal mucosa, which is characterized by symptoms, including sneezing, watery rhinorrhea, nasal obstruction and nasal itching. Eosinophils have long been considered to be the prominent effective cells in allergic inflammation, and eosinophilia has been suggested to favor the development of allergy (3-5). Although the mechanisms underlying the pathogenesis and regulation of AR have been thoroughly investigated, current treatments can only relieve its symptoms. There is currently no treatment method able to cure AR, therefore, additional approaches are required for AR treatment. Genetic therapy offers a promising approach in treating patients with AR.

In response to a variety of stimuli, eosinophils are released from bone marrow to inflammatory tissues through cell surface receptors (4). In particular, the CC chemokine receptor 3 (CCR3), which is a cell-surface guanosine-binding protein-coupled receptor containing a typical motif of seven hydrophobic regions, is primarily expressed on the cell surface of eosinophils. It has been reported that CCR3 is activated in response to eotaxin and contributes to G-protein-dependent intracellular signaling cascades, which leads to the migration of eosinophils $(6,7)$. The importance of CCR3 signaling in allergy was demonstrated previously in studies involving CCR3-deficient mice, which exhibited reduced Th2 responses and an absence of eosinophilia upon allergen sensitization and challenge $(8,9)$. In addition, previous studies have suggested that anti-CCR3 antibody inhibits eosinophil infiltration in animal models and human samples $(10,11)$. Thus, the direct inhibition of CCR3 may serve as a novel approach to effectively alleviate eosinophilia in AR. 
The first case of RNA interference was reported in Caenorhabditis elegans as an endogenous defense mechanism by Fire et al in 1998 (12). RNA interference is an effective gene silencing method, achieved through the transduction of either small interfering RNA (siRNA) or short hairpin RNA (shRNA) (13). Using siRNA or shRNA, rather than oligonucleotide antisense and antibody inhibition, appears to be a more efficient and long-lasting approach to inhibit certain cellular functions due to its ability to target mRNA and affect protein expression in cells (14). Synthetic siRNAs can reduce gene expression, however, this is transient and dose-dependent. By contrast, shRNA can be continuously expressed in cells and then processed by Dicer into siRNA targeting desired genes (15). shRNA carried by a lentivirus can integrate into the host genome and silence gene expression permanently (16). In the present study, the shRNA and lentiviral delivery approach was used for the construction of a mouse CCR3-shRNA-expressing lentiviral vector. CCR3 gene silencing is able to reduce the proliferation of eosinophils and promote eosinophil apoptosis, thereby reducing eosinophil infiltration, and alleviating the symptoms of allergic rhinitis. Therefore, the present study evaluated the effects of this vector on the proliferation and apoptosis of eosinophils.

\section{Materials and methods}

Animals. Male BALB/c mice (5-6-week-old) were maintained on an ovalbumin-free diet under pathogen-free conditions in our animal experimental institute (the Medical Laboratory Animal Center of Nanchang University) at room temperature $\left(22-24^{\circ} \mathrm{C}\right)$ with a 12 -h dark:light cycle. The study protocol was approved by the institutional Animal Care and Use Committees of Nanchang University School of Medicine (Nanchang, China). The present study was performed in accordance with the ethical guidelines of Directive 2010/63/EU (Comments on the European Directive 2010/63/EU for the Protection of Laboratory Animals - see http://ec.europa.eu/environment/chemicals/lab_animals/legislation_en.htm).

Culture of bone marrow-derived eosinophils. The eosinophils originating from bone marrow pluripotent hematopoietic stem cells were collected from the femurs and tibias of wild-type BALB/c mice (Laboratory Animal Centre of Nanchang University School of Medicine), as described previously (17). Briefly, the BALB/c mice were sacrificed by cervical dislocation. The separated femurs and tibias were soaked in $75 \%$ ethanol for $5 \mathrm{~min}$, rinsed with $2 \mathrm{X}$ phosphate-buffered saline (PBS) and then the ends of the femurs and tibias were cut off. The bone marrow was flushed out with Dulbecco's modified Eagle's medium (DMEM) and collected in a plate. A single cell suspension of the bone marrow was obtained by filtering the bone marrow through a syringe with size 7 and size 4 needles. Red blood cell pyrolysis liquid was added to the single cell suspension to eliminate red blood cells, and the single cell suspension was centrifuged at 1,500 rev./min for $10 \mathrm{~min}$. The supernatant was discarded. A cell layer containing eosinophils and eosinophil stem cells was cultured in eosinophil basic culture medium, RPMI 1640 medium (Hyclone; GE Healthcare Life Sciences, Logan, UT, USA), which was supplemented with $20 \%$ fetal bovine serum (FBS; Hyclone; GE Healthcare Life Sciences), 2 mM L-glutamine (Hyclone; GE Healthcare Life Sciences), $50 \mu \mathrm{M} \beta$-mercaptoethanol (Gibco; Thermo Fisher Scientific, Inc., Waltham, MA, USA), $10 \mu \mathrm{g} / \mathrm{ml}$ streptomycin, $100 \mathrm{IU} / \mathrm{ml}$ penicillin (Hyclone; GE Healthcare Life Sciences), $25 \mathrm{mM}$ HEPES, $1 \mathrm{mM}$ sodium pyruvate and $1 \mathrm{X}$ non-essential amino acids (Gibco; Thermo Fisher Scientific, Inc.). Following resuspension, the cells were cultured with the above medium pulsed with $100 \mathrm{ng} / \mathrm{ml}$ FMS-related tyrosine kinase 3 ligand (FLT3-L; PeproTech, Inc., Rocky Hill, NJ, USA) and $100 \mathrm{ng} / \mathrm{ml}$ stem-cell factor (SCF; PeproTech, Inc.) for 4 days. On day 4, $10 \mathrm{ng} / \mathrm{ml}$ recombinant mouse interleukin-5 (rmIL-5; PeproTech, Inc.) was added to replace the FLT3-L and SCF. The cells were then cultured in medium containing rmIL-5 for 10 days. All cell cultures $\left(10^{7} / \mathrm{ml}\right)$ were incubated at $37^{\circ} \mathrm{C}$ in humidified air with $5 \% \mathrm{CO}_{2}$. The eosinophils were identified by hematoxylin and eosin staining for subsequent viral infection.

Constructionofthemurine CCR3(mCCR3)-shRNA-expressing lentiviral vector. The $\mathrm{mCCR} 3$ sequence was obtained from GeneBank (accession no. NM_009914.4). Our previous studies revealed that the formed shuttle plasmid (pLVX-mCCR3-1+2+3+4-shRNA) had a more marked effect on silencing the CCR3 gene $(18,19)$. In the present study, four short tandemly arranged fragments of CCR3 shRNA were subcloned into lentiviruses to form pLVX-mCCR3-1+2+3+4-shRNA (18). This lentiviral vector can deliver a substantial quantity of viral RNA into the DNA of the host cell, and this viral RNA can be then integrated into the DNA of host cells. According to the mCCR3 sequence, four pairs of primers of CCR3 shRNA were designed to amplify four CCR3 shRNA fragments by polymerase chain reaction (PCR). The primer sequences are summarized in Table I. The PCR reaction comprised (in a total volume of $50 \mu \mathrm{l}$ ): $5 \mu \mathrm{l} 10 \mathrm{X}$ short hairpin (sh)DNA annealing buffer, $5 \mu 1$ sense and antisense strands $(100 \mu \mathrm{M})$ and $35 \mu \mathrm{l}$ double distilled $\mathrm{H}_{2} \mathrm{O}$. The PCR cycling steps were $95^{\circ} \mathrm{C}$ for $5 \mathrm{~min} ; 85^{\circ} \mathrm{C}$ for $5 \mathrm{~min} ; 75^{\circ} \mathrm{C}$ for $5 \mathrm{~min}$ and $70^{\circ} \mathrm{C}$ for $5 \mathrm{~min}$; and samples were stored at $4^{\circ} \mathrm{C}$.

pGenesil1.1, pGenesil1.2, pGenesil1.3 and pGenesil1.4 were used as vectors for subcloning the four fragments described above. The restriction enzyme, BsaI (New England BioLabs, Inc., Ipswich, MA, USA), was utilized to cut pGenesil1.1 (bp 2101-2799), pGenesil1.2 (bp 1853-2551), pGenesil1.3 (bp 2103-2801) and pGenesil1.4 (bp 1880-2578). The four fragments of CCR3 shRNA were ligated to the four cut pGenesil vectors using T4DNA ligase separately. The pGenesil vector containing the mCCR3 shRNA was then transformed into recombinant cells, replicated as the recombinant cells proliferated and was extracted from the recombinant cells using a DNA extraction kit. Sequence correct pGenesil-mCCR3-1-shRNA and pGenesil-mCCR3-2-shRNA were cut using HindIII and Bam H I enzymes. The large cut fragment of pGenesil-mCCR3-1-shRNA containing the promoter and mCCR3-1-shRNA was ligated with the small fragment of pGenesil-mCCR3-2-shRNA, (280 bp mCCR3-2-shRNA), to construct pGenesil-mCCR3-1+2-shRNA. Similarly, sequence correct pGenesil-mCCR3-3-shRNA and pGenesil-mCCR3-4-shRNA were cutusingEcoRIandSalI.The large cut fragment of pGenesil-mCCR3-3-shRNA containing 
Table I. Primers for CCR3 short hairpin RNA amplification.

\begin{tabular}{ll}
\hline mCCR3-1F & 5'-CACCGGTTGTGTTGATCCTCATAAATTCAAGAGATTTATGAGGATCAACACAACCTTTTTTG-3' \\
mCCR3-1R & 5'-AGCTCAAAAAAGGTTGTGTTGATCCTCATAAATCTCTTGAATTTATGAGGATCAACACAACC-3' \\
mCCR3-2F & 5'-TTTGGCTGACAATTGACAGATACCTTTCAAGAGAAGGTATCTGTCAATTGTCAGCTTTTTTG-3' \\
mCCR3-2R & 5'-AGCTCAAAAAAGCTGACAATTGACAGATACCTTCTCTTGAAAGGTATCTGTCAATTGTCAGC-3' \\
mCCR3-3F & 5'-CCTCGCAGCATTGCCTGAATTTATCTTCAAGAGAGATAAATTCAGGCAATGCTGCTTTTTTG-3' \\
mCCR3-3R & 5'-AGCTCAAAAAAGCAGCATTGCCTGAATTTATCTCTCTTGAAGATAAATTCAGGCAATGCTGC-3' \\
mCCR3-4F & 5'-TCCCGACCACACCCTATGAATATGATTCAAGAGATCATATTCATAGGGTGTGGTCTTTTTTG-3' \\
mCCR3-4R & 5'-AGCTCAAAAAAGACCACACCCTATGAATATGATCTCTTGAATCATATTCATAGGGTGTGGTC-3' \\
\hline
\end{tabular}

mCCR3, murine CC chemokine receptor 3; F, forward; R, reverse.

the promoter and mCCR3-3-shRNA was ligated with the small cut fragment of pGenesil-mCCR3-4-shRNA (380 bp mCCR3-4-shRNA) to form pGenesil-mCCR3-3+4-shRNA. pGenesil-mCCR3-1+2-shRNA and pGenesil-mCCR3$3+4$-shRNA were cut using BamH I and SalI. The large cut fragment of pGenesil-mCCR3-1+2-shRNA containing the promoter and mCCR3-1+2-shRNA was ligated with the small cut fragment of pGenesil-mCCR3-3+4-shRNA to form pGenesil-mCCR3-1+2+3+4-shRNA. To construct the lentiviral mCCR3-1+2+3+4-shRNA, a pLVX-shRNA2-m lentiviral vector was used. The pLVX-ShRNA2-m vector (Biowit Technologies Ltd., Quincy, MA, USA) was cut using MluI and XhoI enzymes. pGenesil-mCCR3-1+2+3+4-shRNA were cut using MulI and SalI enzymes. The large fragment of the cut pLVX-shRNA2-m containing a promotor was ligated with the small fragment of the cut pGenesil-mCCR3-1+2+3+4-shRNA, to form the target plasmid, pLVX-mCCR3-1+2+3+4-shRNA. Validation of the sequences of mCCR3-1+2+3+4-shRNA were confirmed using DNA sequencing and are shown in Table II.

Packaging of the pLVX-ShRNA2-m vector and the constructed $p L V X-m C C R 3-1+2+3+4-$ shRNA plasmid, and the culture of eosinophils with viral infection. To ensure the safety and the titer of the pLVX-ShRNA2-m vector and the constructed pLVX-mCCR3-1+2+3+4-shRNA, these two lentiviruses were separately co-transfected with the packaging plasmids, Baculo p35, pCMV R8.2 and VSV (quantities: $2 \mu \mathrm{g}$ Baculo p35, $2 \mu \mathrm{g}$ VSV plasmid, $4.7 \mu \mathrm{g}$ pCMV R8.2 plasmid and $2.3 \mu \mathrm{g}$ Lentiviral vector), into $293 \mathrm{~T}$ cells in $100-\mathrm{mm}$ tissue culture dishes of DMEM containing $10 \% \mathrm{FBS}$ without antibiotics at $37^{\circ} \mathrm{C}$. The medium was replaced $24 \mathrm{~h}$ later, and the virus-containing medium was harvested $48 \mathrm{~h}$ following transduction. The supernatants were filtered through a $0.22 \mu \mathrm{m}$ syringe filter (EMD Millipore, Billerica, MA, USA). The eosinophil cultures on day 10 were infected with the supernatants at a multiplicity of infection of 50, and polybrene (Sigma-Aldrich; Thermo Fisher Scientific, Inc.) was added to a final concentration of $8 \mu \mathrm{g} / \mathrm{ml}$. The harvested eosinophils were transfected with either a blank control (RPMI 1640 medium), empty vector (pLVX-shRNA2-m) or the constructed target plasmid (pLVX-mCCR3-1+2+3+4-shRNA), respectively. The culture medium was aspirated $48 \mathrm{~h}$ following transduction and the cells were washed with PBS for the subsequent quantitative
Table II. Sequences of four shRNAs for plasmid construction

$\begin{array}{ll}\text { mCCR3-1 shRNA } & \text { 5'-GGTTGTGTTGATCCTCATAAA-3' } \\ \text { mCCR3-2 shRNA } & \text { 5'-GCTGACAATTGACAGATACCT-3' } \\ \text { mCCR3-3 shRNA } & \text { 5'-GCAGCATTGCCTGAATTTATC-3' } \\ \text { mCCR3-4 shRNA } & \text { 5'-GACCACACCCTATGAATATGA-3 }\end{array}$

mCCR3, murine CC chemokine receptor 3; shRNA, short hairpin RNA.

(q)PCR and western blot analyses, and terminal deoxynucleotidyl transferase dUTP nick end labeling (TUNEL) and 3-(4,5-dimethylthiazol-2-yl)-5-(3-carboxymethoxy phenyl)-2(4-sulfophenyl)-2H-tetrazolium (MTT) assays.

qPCR analysis. The eosinophils were suspended in TRIzol (Invitrogen; Thermo Fisher Scientific, Inc.) and RNA was extracted according to the manufacturer's protocol. The RNA was converted into cDNA using a High Capacity cDNA Reverse Transcription kit (Applied Biosystems; Thermo Fisher Scientific, Inc.) according to the manufacturer's protocol. The cDNA was used with SYBR ${ }^{\circledR}$ Green qPCR SuperMix (Invitrogen; Thermo Fisher Scientific, Inc.). For the detection of CCR3, GAPDH was used as a control. The primers of CCR3 (ID: NM_009914.4) and GAPDH (ID: NM_017008.4) were designed as follows: CCR, forward 5'-CTG GCA CAC AGA CCC TAG AA-3' and reverse 5'-TTG AGT CTC TGA ACG CAT CA-3'; and GAPDH, forward 5'-GGC CTC CAA GGA GTA AGA AA-3' and reverse 5'-GCC CCT CCT GTT ATT ATG G-3'. The total reaction mixture was run on a 7500 Real-Time system (Applied Biosystems; Thermo Fisher Scientific, Inc.) with relative quantitation according to the manufacturer's protocol. The following thermocycling steps were used: $95^{\circ} \mathrm{C}$ denaturation for $10 \mathrm{sec}$, one cycle; $95^{\circ} \mathrm{C}$ denaturation for $5 \mathrm{sec} ; 54^{\circ} \mathrm{C}$ annealing extension for $30 \mathrm{sec}$, a total of 40 cycles; $95^{\circ} \mathrm{C}$ for $1 \mathrm{~min}$, one cycle, and $55^{\circ} \mathrm{C}$ for $30 \mathrm{sec}, 41$ cycles.

Western blot analysis. The eosinophils were homogenized in RIPA lysis buffer (Pierce Biotechnology, Inc., Rockford, IL, USA). After $20 \mathrm{~min}$ on ice, insoluble materials were removed by centrifugation at $4^{\circ} \mathrm{C}$ at $14,000 \mathrm{x} g$. The supernatants were mixed with SDS sample buffer and boiled for 
A
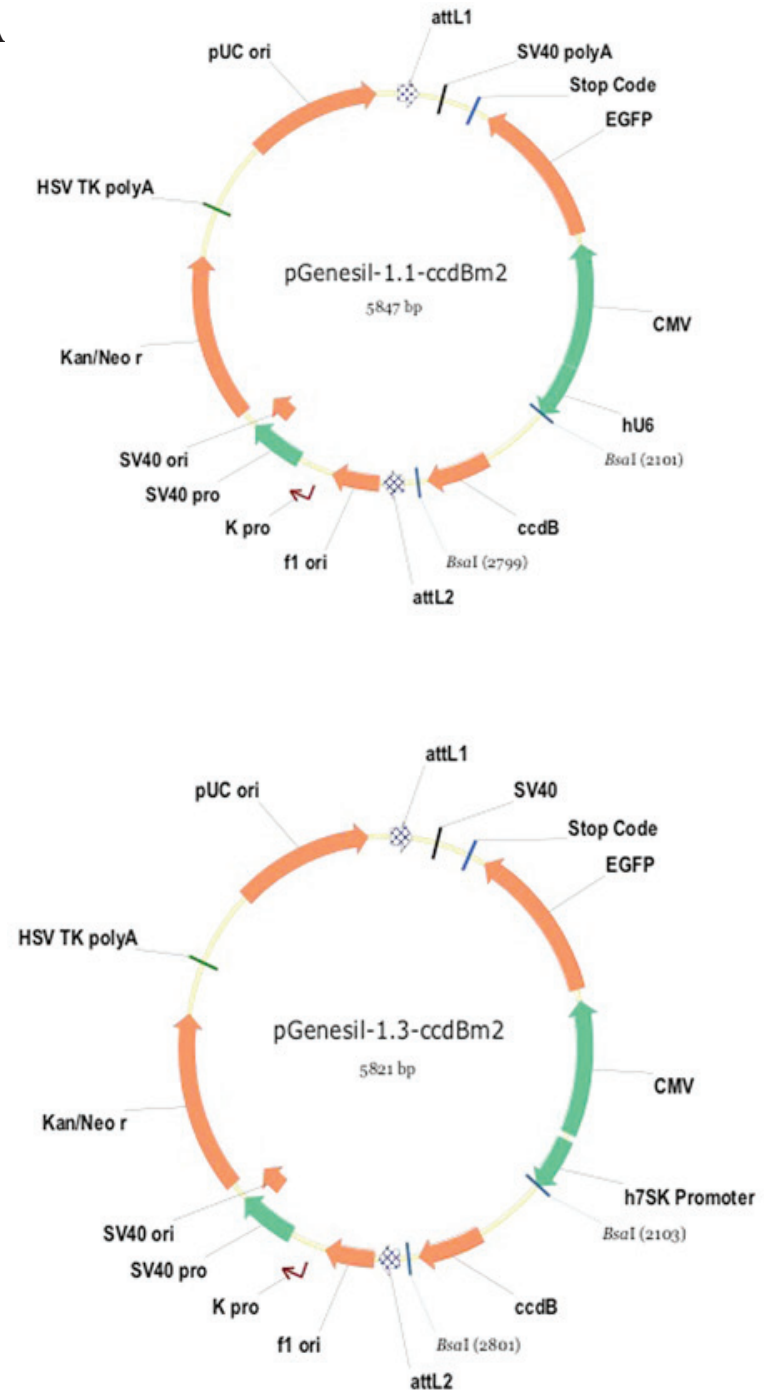
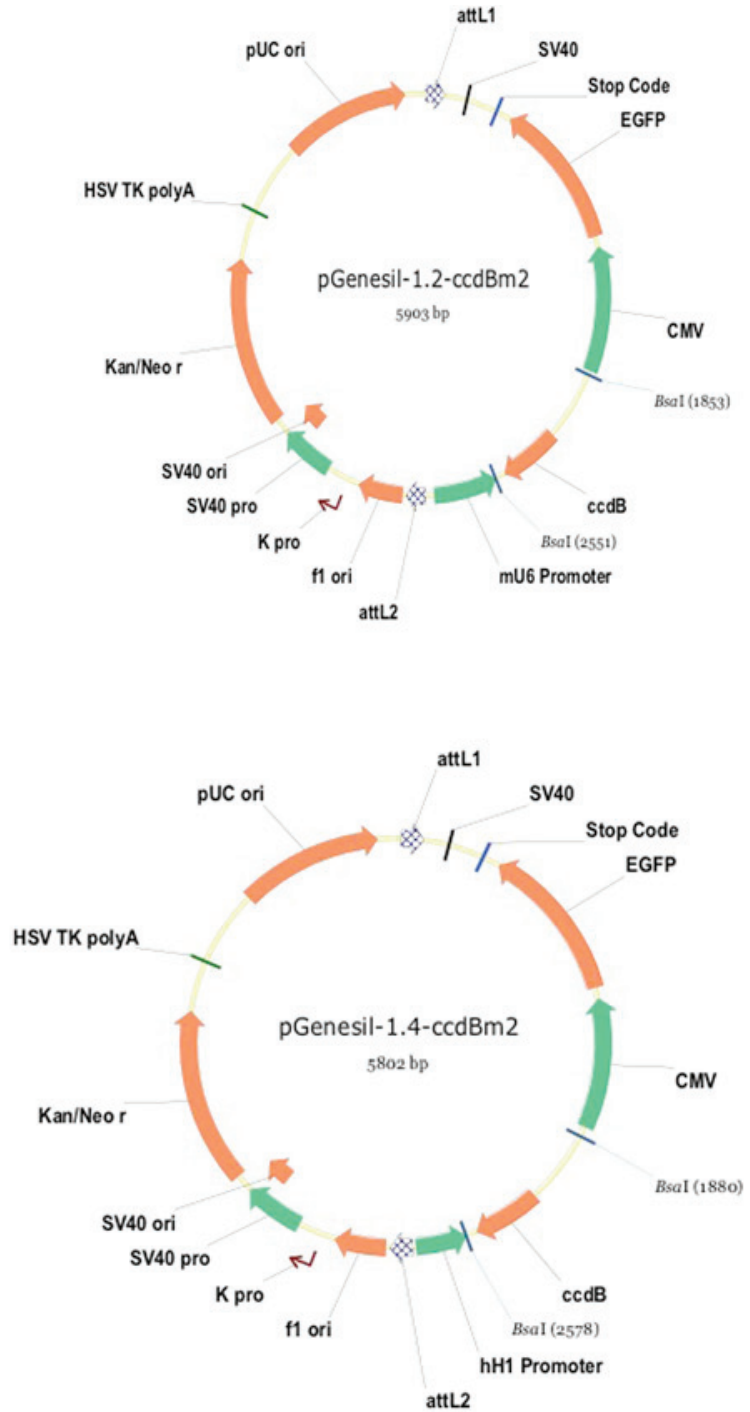

B

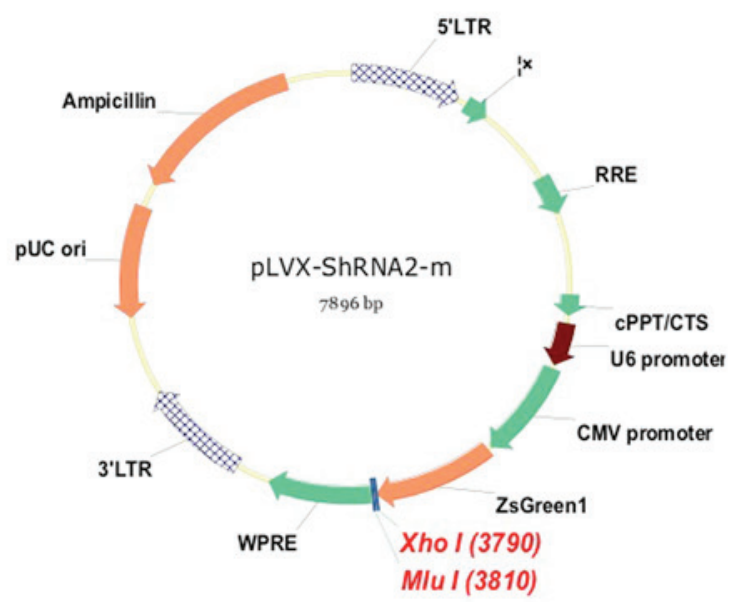

Figure 1. (A) Structures of pGenesil vectors. (B) Schematic structure for engineering of the pLVX lentiviral vector. A four-tandem-fragment of CCR3-1+2+3+4-shRNA was inserted between aa3790 and aa3810 to form the pLVX-CCR3-1+2+3+4-shRNA plasmid, which was ampicillin resistant and carried the green fluorescent protein gene. CCR3, murine CC chemokine receptor 3; shRNA, short hairpin RNA.

$5 \mathrm{~min}$. The proteins were separated on SDS-polyacrylamide $(10 \%)$ gels, following which they were blotted onto PVDF membranes (EMD Millipore). Non-specific protein binding sites were blocked by incubation with $5 \%$ bovine serum albumin in TBST buffer $(20 \mathrm{mM}$ Tris- $\mathrm{HCl}, 137 \mathrm{mM} \mathrm{NaCl}$ and $0.05 \%$ Tween 20) at $\mathrm{pH} 7.6$ for $1 \mathrm{~h}$, followed by incubation with rabbit polyclonal anti-CCR3 primary antibody (cat. no. AJ1417a; 1:200 dilution; Santa Cruz Biotechnology, Inc.) overnight at $4^{\circ} \mathrm{C}$. The membranes were washed three times with TBST, followed by incubated with horseradish 
peroxidase-conjugated goat anti-rabbit IgG secondary antibody (cat. no. C1508; 1:20,000 dilution; SouthernBiotech, Burmingham, AL, USA) for $1 \mathrm{~h}$ at room temperature. The blots were visualized using an enhanced chemiluminescence system (GE Healthcare Life Sciences) according to the manufacturer's protocol. To normalize for protein content, the blots were stripped and stained with GAPDH antibody (cat. no. KC-5G5; 1:10,000 dilution; Abcam, Cambridge, MA, USA) overnight at $4^{\circ} \mathrm{C}$. The concentration of each target protein was normalized against that of GAPDH.

TUNEL assay. Quantitative assessment of apoptosis in the eosinophils was performed using a TUNEL method according to the manufacturer's protocol (Promega, Madison, WI, USA). Briefly, the eosinophils were incubated with either the blank control (RPMI 1640 medium), empty vector (pLVX-shRNA2-m) or the constructed target plasmid (pLVX-mCCR3-1+2+3+4-shRNA), respectively, for $48 \mathrm{~h}$. The cells were then trypsinized, fixed with $4 \%$ paraformaldehyde and permeabilized with $0.1 \%$ Triton-X-100 in $0.1 \%$ sodium citrate. Following washing with PBS, the cells were incubated with the reaction mixture for $60 \mathrm{~min}$ at $37^{\circ} \mathrm{C}$. The stained cells were then analyzed using a FACScan cytometer (BD Biosciences, Franklin Lakes, NJ, USA).

Cell proliferation assay. The eosinophils were plated at a density of $1 \times 10^{5}$ per well $(100 \mu \mathrm{l})$ in 96-well plates and treated with either blank control (RPMI 1640 medium), empty vector (pLVX-shRNA2-m) or the constructed target plasmid (pLVX-mCCR3-1+2+3+4-shRNA) for different durations $(0,24,48$ and $72 \mathrm{~h})$. The culture media were then removed and the cells were washed with PBS. An MTS assay was performed using a kit from Promega in accordance with the manufacturer's protocol. The absorbance was measured at a fixed wavelength of $490 \mathrm{~nm}$ on a microplate reader (VersaMax; Molecular Devices, LLC, Sunnyvale, CA, USA). Each data point was normalized to the value of their corresponding control samples.

Statistical analysis. The results obtained from the blank control, empty vector (pLVX-shRNA2-m) and constructed target plasmid (pLVX-mCCR3-1+2+3+4-shRNA) groups were analyzed using one-way analysis of variance using SPSS 18 software (SPSS, Inc., Chicago, IL, USA). Each experiment was repeated three times. Data are presented as the mean \pm standard error of the mean of triplicate samples. $\mathrm{P}<0.05$ was considered to indicate a statistically significant difference.

\section{Results}

Construction of the mCCR3-shRNA plasmid. As described in the previous section, mCCR $3-1+2+3+4$-shRNA, the sequence of which was confirmed using DNA sequencing, was successfully ligated using a sub-cloning technique with the pGenesil1 vectors, pGenesil1.1, pGenesil1.2, pGenesil1.3 and pGenesil1.4 (Fig. 1A). The recombinant fragment of the mCCR3-1+2+3+4-shRNA was successfully inserted into the pLVX-shRNA2-m lentiviral vector, to obtain the pLVX-CCR3-1+2+3+4-shRNA vector (Fig. 1B). This novel
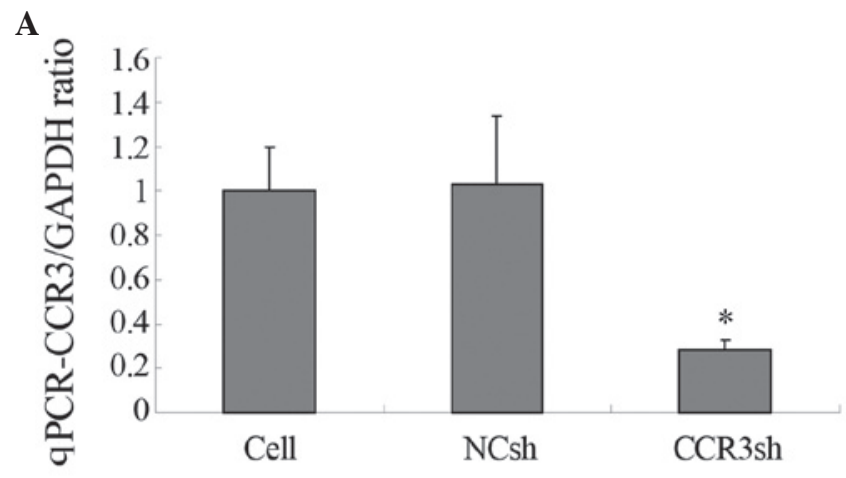

B

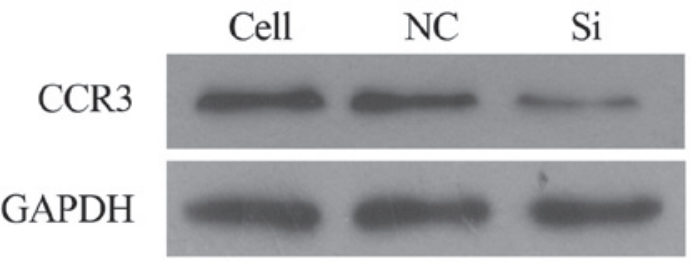

Figure 2. Expression of CCR3. (A) qPCR determination of cellular CCR3/GAPDH ratio. The expression of CCR3mRNA was significantly inhibited by pLVX-shRNA2-mCCR3-1+2+3+4shRNA, but not by the NCsh vector $\left({ }^{*} \mathrm{P}<0.05\right)$. (B) Expression of CCR3, determined using western blot analysis. GAPDH, a house-keeping protein was used as a control. The expression of CCR3 was significantly inhibited by pLVX-shRNA2-mCCR3-1+2+3+4shRNA, but not by NC. CCR3, murine CC chemokine receptor 3; shRNA, short hairpin RNA; Cell, blank control (cells only); NCsh/NC, cells transfected with empty vector; CCR3sh/Si, cells transfected with pLVX-shRNA2-mCCR3-1+2+3+4shRNA; qPCR, quantitative polymerase chain reaction.

lentiviral vector was then packaged into 293T cells by co-transfection with the Baculo p35, pCMV R8.2 and VSV packaging plasmids. In a pilot experiment, the highest transduction efficiency of the pLVX-mCCR3-1+2+3+4-shRNA virus was observed at a multiplicity of infection of 10 .

Detection of the expression levels of $m C C R 3$ in eosinophils. The preliminary experiment demonstrated that the pLVX-mCCR3-1+2+3+4-shRNA, which contained four different interfering shRNAs against mCCR3, had higher gene silencing efficiency, compared with any single shRNA of mCCR 3 in the eosinophils, determined using qPCR. As shown in Fig. 2A, the mRNA level of $m C C R 3$ was significantly inhibited only by transduction with pLVX-mCCR3-1+2+3+4-shRNA. The mRNA levels of mCCR3 were not affected by transduction with the negative control shRNA vector (Fig. 2A). In addition, the protein level of mCCR3 was markedly inhibited by transduction with pLVX-mCCR3-1+2+3+4-shRNA, as determined using western blot analysis. Similarly, the protein expression of mCCR 3 was not altered by transduction with the negative control shRNA vector (Fig. 2B).

Silencing ofmCCR 3 withlentiviral shRNA promotes apoptosis of eosinophils. To investigate whether the downregulation of mCCR3 with lentiviral shRNA can induce apoptosis of eosinophils, the TUNEL method was used. The results showed that $<8 \%$ of the eosinophils showed apoptosis in the blank control- and empty vector-transduced cells. However, $45 \%$ of the pLVX-mCCR3-1+2+3+4shRNA-transduced eosinophils 
A

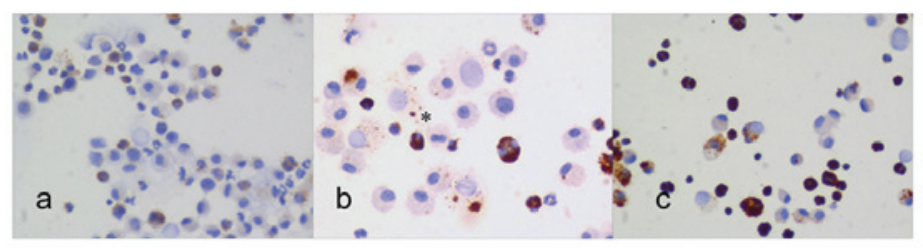

$\mathbf{B}$

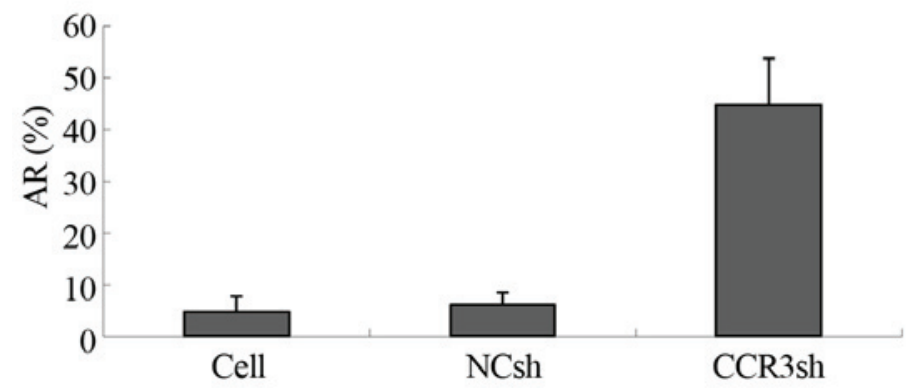

Figure 3. (A) Terminal deoxynucleotidyl transferase dUTP nick end labeling assay of eosinophil apoptosis. Cells with brown nuclei are apoptotic. (a) blank control cells; (b) cells with empty vector; (c) cells with pLVX-shRNA2-mCCR3-1+2+3+4shRNA. (B) CCR3shRNA promoted cell apoptosis in vitro. pLVX-shRNA2-mCCR3-1+2+3+4shRNA induced $~ 45 \%$ apoptosis of cells. CCR3, murine CC chemokine receptor 3; shRNA, short hairpin RNA; AR, apoptotic rate; Cell, blank control (cells only); NCsh/NC, cells transfected with empty vector; CCR3sh/Si, cells transfected with pLVX-shRNA2-mCCR3-1+2+3+4shRNA

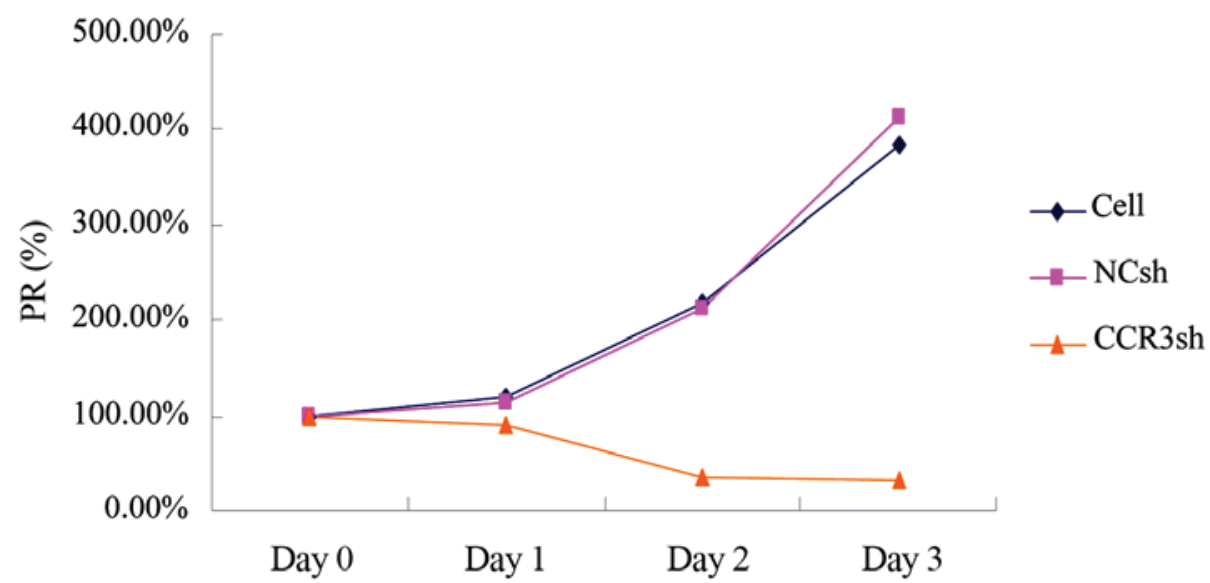

Figure 4. 3-(4,5-dimethylthiazol-2-yl)-5-(3-carboxymethoxyphenyl)-2-(4-sulfophenyl)-2H-tetrazolium assay of eosinophil proliferation. CCR3, murine CC chemokine receptor 3; shRNA, short hairpin RNA; PR, proliferation rate; Cell, blank control, cells only; NCsh: cells transfected with empty vector; CCR3sh: cells transfected with pLVX-shRNA2-mCCR3-1+2+3+4shRNA.

exhibited apoptotic characteristics (Fig. 3A and B). This result suggested that expression of mCCR3 was critical to the survival of the eosinophils.

Silencing of $m C C R 3$ with lentiviral shRNA reduces the proliferation of eosinophils. The present study used an MTS assay to investigate the effect of mCCR3-shRNA on the proliferation of eosinophils. The cells were incubated with MTS reagents $0,24,48$ and 72 post-transduction with virons carrying pLVX-mCCR $3-1+2+3+4$-shRNA. As shown in Fig. 4, transduction with pLVX-mCCR3-1+2+3+4-shRNA started to inhibit cell proliferation at $24 \mathrm{~h}(80 \%)$, and the cell proliferation rate decreased to $20 \%$ at $48 \mathrm{~h}$ and to $19 \%$ at $72 \mathrm{~h}$ (Fig. 4). By contrast, cell proliferation rates in the blank control group and empty vector control group were increased, reaching $200 \%$ at $48 \mathrm{~h}$ and $400 \%$ at $72 \mathrm{~h}$ (Fig. 4).

\section{Discussion}

Eosinophils are considered to be a critical factor in the induction of inflammation and allergy by releasing reactive oxygen species and cytotoxic molecules, including major basic protein, eosinophilic peroxidase, eosinophil-derived neurotoxin and eosinophil cationic protein (5). Eosinophils develop from CD34 ${ }^{+}$ hematopoietic progenitor cells within the bone marrow under the stimulation of cytokines, including granulocyte-macrophage colony-stimulating factor (GM-CSF), IL-3 and IL-5 (20). IL-5 is predominantly expressed in white blood cells and is a key modulatory cytokine, which is important in regulating the proliferation, differentiation and activation of eosinophils (21). Allergic IL-5-deficiency in mice leads to reduced numbers of eosinophilia in the bone marrow and blood, and eosinophils are recruited to the tissue in reduced numbers in response to 
allergen exposure. However, treating patients with anti-IL-5 monoclonal antibody only partially reduced eosinophilia in airway tissues and bone marrow $(22,23)$, suggesting that other factors contribute to eosinophil survival in these tissues. With the exception of IL-5, GM-CSF and IL-3 have also been known to have growth factor effects on eosinophils (20). The present study revealed that knockdown of CCR3 by specific shRNA efficiently inhibited eosinophil proliferation and promoted eosinophil apoptosis. Although the mechanism underlying these effects were not investigated, there are several possible mechanisms. The FBS used in the culture medium contains the eosinophil-associated growth-factors IL-5, IL-3 and GM-CSF. As CCR3 protein was expressed in the eosinophils in control group, IL-5-, IL-3- and GM-CSF-induced eosinophil growth may require CCR3 in its growth signaling pathway. When CCR3 was silenced by CCR3 shRNA, the growth pathway involving IL-5, IL-3 and GM-CSF was not active, therefore, the proliferation rate of the eosinophils declined rapidly. In terms of why silencing CCR3 resulted in apoptosis, CCR3 may be associated with factors involved in the apoptotic signaling pathway, including p53, p73, B cell lymphoma-2-associated X protein (BAX), phorbol-12-myristate-13-acetate-induced protein 1 (Noxa) and p53 upregulated modulator of apoptosis (PUMA). The present study hypothesized that CCR3 inhibits the above factors and inhibits apoptosis, and when CCR3 was silenced by CCR3 shRNA, the above factors in the apoptotic signaling pathway were activated, causing eosinophils to undergo apoptosis.

In conclusion, using the techniques described, pLVX-mCCR3-1+2+3+4shRNA was successfully constructed in the present study. The results demonstrated that virions pLVX-mCCR $3-1+2+3+4$ shRNA significantly reduced the mRNA and protein expression levels of CCR3, promoted eosinophil apoptosis and inhibited eosinophil proliferation. This may have contributed to the inhibition of eosinophil infiltration in the airway. However, the mechanism underlying the eosinophil apoptosis and proliferation inhibition induced by CCR3 silencing requires further investigation. An understanding of the fundamental causes of regulating eosinophil apoptosis may lead to novel strategies for the treatment of allergic inflammation. Subsequent investigations aim to use a single shRNA-expressing lentiviral vector targeting IL-5 and CCR3 to affect eosinophil infiltration in the airway tissues in vitro and in vivo.

\section{Acknowledgements}

The authors would like to thank the Molecular Biology Center in Jiangxi province for its continuing support. This study was supported by grants from the National Natural Science Foundation of China (grant no. 81060084), the Jiangxi Provincial Natural Science Foundation (grant no. 2010GZY0251) and the Jiangxi Provincial Department of Science and Technology project (grant no. 20133BBG70071).

\section{References}

1. Brozek JL, Bousquet J, Baena-Cagnani CE, Bonini S Canonica GW, Casale TB, van Wijk RG, Ohta K, Zuberbier T and Schünemann HJ: Allergic rhinitis and its impact on asthma (ARIA) guidelines: 2010 revision. J Allergy Clin Immunol 126 466-476, 2010.
2. Zhang Y and Zhang L: Prevalence of allergic rhinitis in China. Allergy Asthma Immunol Res 6: 105-113, 2014.

3. Plaut M and Valentine MD: Clinical practice. Allergic rhinitis. N Engl J Med 353: 1934-1944, 2005.

4. Weller PF: The immunobiology of eosinophils. N Engl J Med 324: 1110-1118, 1991.

5. Stone KD, Prussin C and Metcalfe DD: IgE, mast cells, basophils, and eosinophils. J Allergy Clin Immunol 125 (2 Suppl 2): S73-S80, 2010.

6. Ponath PD, Qin S, Post TW, Wang J, Wu L, Gerard NP, Newman W, Gerard C and Mackay CR: Molecular cloning and characterization of a human eotaxin receptor expressed selectively on eosinophils. J Exp Med 183: 2437-2448, 1996.

7. Sallusto F, Mackay CR and Lanzavecchia A: Selective expression of the eotaxin receptor CCR3 by human T helper 2 cells. Science 277: 2005-2007, 1997.

8. Fulkerson PC, Fischetti CA, McBride ML, Hassman LM, Hogan SP and Rothenberg ME: A central regulatory role for eosinophils and the eotaxin/CCR3 axis in chronic experimental allergic airway inflammation. Proc Natl Acad Sci USA 103: 16418-16423, 2006.

9. Pope SM, Zimmermann N, Stringer KF, Karow ML and Rothenberg ME: The eotaxin chemokines and CCR3 are fundamental regulators of allergen-induced pulmonary eosinophilia. J Immunol 175: 5341-5350, 2005.

10. Chuang CC, Su KE, Chen CW, Fan CK, Lin FK, Chen YS and Du WY: Anti-CCR3 monoclonal antibody inhibits eosinophil infiltration in Angiostrongylus cantonensis-infected ICR mice. Acta Trop 113: 209-213, 2010.

11. Heath H, Qin S, Rao P, Wu L, LaRosa G, Kassam N, Ponath PD and Mackay CR: Chemokine receptor usage by human eosinophils. The importance of CCR3 demonstrated using an antagonistic monoclonal antibody. J Clin Invest 99: 178-184, 1997.

12. Fire A, Xu S, Montgomery MK, Kostas SA, Driver SE and Mello CC: Potent and specific genetic interference by double-stranded RNA in Caenorhabditis elegans. Nature 391: 806-811, 1998.

13. DeVincenzo JP: The promise, pitfalls and progress of RNA-interference-based antiviral therapy for respiratory viruses. Antivir Ther 17: 213-225, 2012.

14. Karimi MH, Ebadi P, Pourfathollah AA, Moazzeni M, Soheili ZS and Samiee S: Comparison of three techniques for generation of tolerogenic dendritic cells: siRNA, oligonucleotide antisense and antibody blocking. Hybridoma (Larchmt) 29: 473-480, 2010.

15. Paddison PJ, Caudy AA, Bernstein E, Hannon GJ and Conklin DS: Short hairpin RNAs (shRNAs) induce sequence-specific silencing in mammalian cells. Genes Dev 16: 948-958, 2002.

16. Rubinson DA, Dillon CP, Kwiatkowski AV, Sievers C, Yang L, Kopinja J, Rooney DL, Zhang M, Ihrig MM, McManus MT, et al: A lentivirus-based system to functionally silence genes in primary mammalian cells, stem cells and transgenic mice by RNA interference. Nat Genet 33: 401-406, 2003.

17. Dyer KD, Moser JM, Czapiga M, Siegel SJ, Percopo CM and Rosenberg HF: Functionally competent eosinophils differentiated ex vivo in high purity from normal mouse bone marrow. J Immunol 181: 4004-4009, 2008.

18. Zhu XH, Liao B, Wang XY, Liu K and Liu YH: Construction and identification of mouse eosinophils CCR3gene RNA interference lentiviral vector. Zhonghua Er Bi Yan Hou Tou Jing Wai Ke Za Zhi 48: 316-321, 2013.

19. Zhu XH, Liao B, Liu K and Liu YH: Effect of RNA interference therapy on the mice eosinophils CCR3 gene and granule protein in the murine model of allergic rhinitis. Asian Pac J Trop Med 7: 226-230, 2014.

20. Tai PC, Sun L and Spry CJ: Effects of IL-5, granulocyte/macrophage colony-stimulating factor (GM-CSF) and IL-3 on the survival of human blood eosinophils in vitro. Clin Exp Immunol 85: 312-316, 1991.

21. Coffman RL, Seymour BW, Hudak S, Jackson J and Rennick D: Antibody to interleukin-5 inhibits helminth-induced eosinophilia in mice. Science 245: 308-310, 1989.

22. Cho JY, Miller M,Baek KJ,Han JW, Nayar J, Lee SY, McElwain K, McElwain S, Friedman S and Broide DH: Inhibition of airway remodeling in IL-5-deficient mice. J Clin Invest 113: 551-560, 2004.

23. Leckie MJ, ten Brinke A, Khan J, Diamant Z, O'Connor BJ, Walls CM, Mathur AK, Cowley HC, Chung KF, Djukanovic R, et al: Effects of an interleukin-5 blocking monoclonal antibody on eosinophils, airway hyper-responsiveness, and the late asthmatic response. Lancet 356: 2144-2148, 2000. 